\title{
Advanced Silicate-based Lubricant Additive Induced Diamond-like Carbon \\ Structured Restoration Layer
}

Jing Zhang*1, Yanhong $\mathrm{Gu}^{2}$, Jiajun $\mathrm{Liu}^{3}$

Department of Mechanical Engineering,

1. Indiana University - Purdue University Indianapolis, IN 46202, USA

2. Department of Mechanical Engineering,

University of Alaska Fairbanks, Fairbanks, AK 99775, USA

3. Tsinghua University, Beijing 100084, China

*corresponding author: Tel: 317-278-7186; E-mail: jz29@iupui.edu

\begin{abstract}
An advanced silicate based lubricant additive has been employed in long-term pin-ondisk tribological experiments. The worn steel/steel surfaces were characterized using nano-indentation, SEM, XPS, and Raman spectroscopy for their physical, mechanical, and chemical properties. The average nano-hardness of the repaired layers on the disk and the pin is $10.2 \mathrm{GPa}$ and $16.7 \mathrm{GPa}$ respectively, which is substantially higher than that of the disk (HV 221, or $0.71 \mathrm{GPa}$ ) and the pin (HRC55, or 1.8 GPa) before tribological tests, forming super hard surfaces on the contact pair surfaces. Combined Raman spectroscopy and XPS studies suggest the formation of diamond-like carbon based restoration layers. A new formation mechanism of the restoration DLC layer contributing to hard and smooth contact surfaces is proposed.
\end{abstract}

Keywords: Lubricant additives; Diamond-like carbon; Restoration layer; Raman spectroscopy

This is the author's manuscript of the article published in final edited form as: Zhang, J., Gu, Y., Liu, J. (2015). Advanced silicate-based lubricant additive induced diamond-like carbon structured restoration layer. Tribology International 90: 263-269. http://dx.doi.org/10.1016/j.triboint.2015.04.036 


\section{Introduction}

Modern machining process conditions have become increasingly demanding, which typically involve elevated temperature, high pressure, and high velocity. Lubrication has a long history as an effective means to improve wear resistance and reduce friction [1-5]. An advanced silicate-based lubricant additive $\mathrm{Al}_{4}\left[\mathrm{Si}_{4} \mathrm{O}_{10}\right](\mathrm{OH})_{4}[6,7]$ has been developed recently. The most noteworthy feature of the additive is that only a small amount $(<1 \mathrm{wt} \%)$ can significantly reduce friction, and even restore the friction pair surface. Several studies have shown that the additive substantially improved the anti-wear and friction-reducing properties of steel-steel friction pairs [810]. Yu [11] investigated the restoration layer formed on the worn surface using the lubricant additive composed of flaky aluminum-magnesium silicate and catalysts. It was reported that a diamond-like carbon (DLC) film with Si or Si-O elements and high hardness formed on the worn surface [11]. Although the above studies, there is no study under long-term operating conditions. In addition, most published research mainly focused on the friction performance of restoration layer, but confirmation of DLC layer using Raman analysis and the role of lubricant additives on the formation of DLC layer have not yet been reported.

The purpose of this study is to understand the effect of the silicate-based additive in long-term 300-hour tribological tests. The worn surfaces with the application of lubricant additive will be characterized. The formation of DLC layer on the repaired contact layer will be examined by Raman spectroscopy. The DLC formation mechanism will also be proposed. It should be noted that the diamond-like carbon structure in the work is different from conventional DLC film which is widely 
accepted as a kind of amorphous carbon film material prepared by CVD/PVD and liquid phase methods.

\section{Experimental details}

\subsection{Tribological tests}

Tests were performed using a pin-on-disk tribo-tester. It has 45 steel pins and disks with a chemical composition of $0.42-0.50$ wt\% C, $0.17-0.37$ wt\% Si, $0.50-0.80$ wt\% $\mathrm{Mn}, \leq 0.2 \mathrm{wt} \% \mathrm{Cr}, \leq 0.30 \mathrm{wt} \% \mathrm{Ni}$ and $\leq 0.25 \mathrm{wt} \% \mathrm{Cu}$ with $\mathrm{Fe}$ as the balance. The dimension of 45 steel pin was $8 \mathrm{~mm}$ in thickness and diameter $12.7 \mathrm{~mm}$, and the disk was $70 \mathrm{~mm}$ in diameter and $3 \mathrm{~mm}$ in thickness. The pins and the disks were controlled to be HRC55 and HV 221 hardness by heat treatment respectively. Each specimen was finished to a constant surface roughness of $0.8 \mu \mathrm{m}$ Ra.

Continuous 300-hour test with a variational loading profile 98 N (0-72h), 147 N (72156h) and $245 \mathrm{~N}$ (156-300 h) was carried out to discover the properties of the worn surface.

\subsection{Lubricant and additive}

The lubricant used in this experiment was SAE 30 engine oil as a baseline. We did not use additive-free base oil because we try to study the tribological properties in a simulated realistic condition. Silicate-based lubricant $\mathrm{Al}_{4}\left[\mathrm{Si}_{4} \mathrm{O}_{10}\right](\mathrm{OH})_{4}$ was used as additional additive. The additive is composed of silicate powders prepared from natural minerals and some dispersants with an average particle size of $0.3-3 \mu \mathrm{m}[6$, 12]. The additive was added to the SAE 30 engine oil at a concentration of $0.8 \mathrm{wt} \%$. 
During the tests, the lubricant oil was fed into the contact point between the pin and the disk by a circulating drip-feeding pipe. The average feeding rate of the flow was about $10 \mathrm{ml} / \mathrm{min}$.

\subsection{Worn surface analysis}

After the tribological tests, atomic force microscope (AFM, SPM-9500J2; Shimadzu, Tokyo, Japan) was used to measure the roughness. A nano-indenter (ENT-1100, Elionix Co., Tokyo, Japan) was used to measure the nano-hardness of the worn surface, in which the samples were indented with maximum applied loads in the range of $0.5-500 \mathrm{mN}$. For each sample, the hardness was calculated using five tests.

To characterize the microstructure and wear mechanism of the coated samples, SEM (JSM-6460LV; JEOL Co. Ltd., Japan) was used to observe the cross-sectional morphology of the repaired pin in 300-hour test.

The samples were irradiated with Raman spectroscopy (RS; RM2000, Renishaw, England) to study the DLC structure of the samples. The measurements were carried out with a laser wavelength of $514.532 \mathrm{~nm}$. A low input power of $3.5 \mathrm{~mW}$ was used in order to minimize possible beam-heating effects. The structural characterization of DLC film formed on the worn surfaces was performed using X-ray photoelectron spectroscopy (XPS, AEM PHI5300, PE Co.).

\section{Results}

3.1 The roughness and nano-hardness of the worn surface 
Surface roughness of the disk and the pin was measured using an AFM with two scan areas. The original surface roughness Ra of the samples was about $0.8 \mu \mathrm{m}$, whereas that of two different scan areas of the worn surface became $\mathrm{Ra}=60.318 \mathrm{~nm}$ on the disk), $8.053 \mathrm{~nm}$ on the pin. It can be considered that the surface roughness was significantly reduced in the friction and wear process.

The average results of nano-hardness at five different locations on the worn surfaces are shown in Fig.1. The depth of indentation in the nano-hardness test was $150 \mathrm{~nm}$, indicating the hardness of the repaired layer. The average nano-hardness of the repaired layers on the disk and the pin is 10.2 GPa (Fig. 1a) and 16.7 GPa (Fig. 1b) respectively. The hardness values are substantially higher than the hardness of the disk (HV 221, or $0.71 \mathrm{GPa}$ ) and the pin (HRC55, or $1.8 \mathrm{GPa}$ ) before the tribological tests, suggesting forming super hard surfaces on the contact pair surfaces. It should also be mentioned that the nano-hardness of the worn surface on the pin was $50 \%$ greater than that on the disk, suggesting the running time influenced the mechanical properties of the repaired layer significantly. It was also noted that the nano-hardness data of the worn disk was more scattered than that of the worn pin, which indicated, the effect of the silicate additive possessed certain selectivity at different locations of the worn surface [13]. 

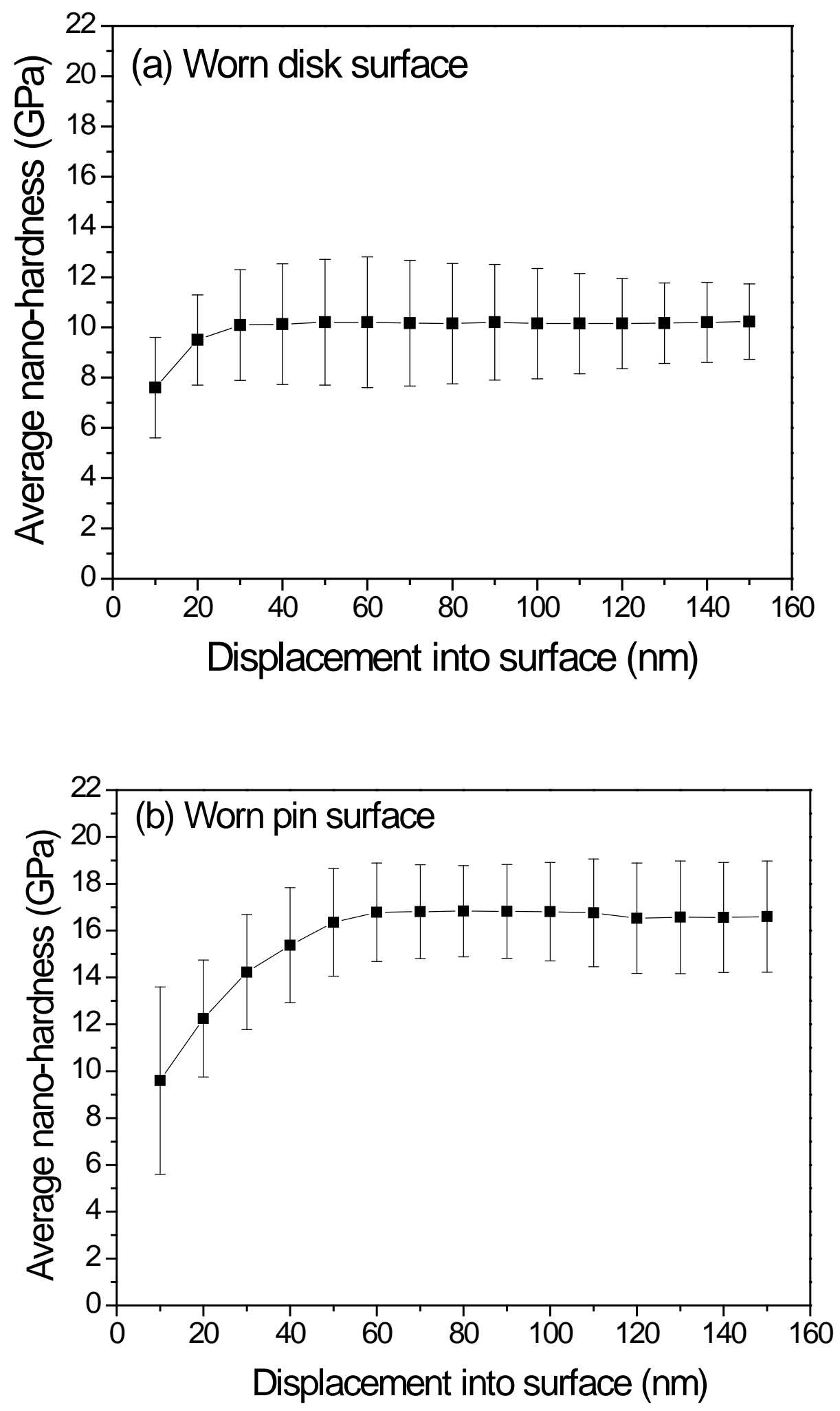

Fig. 1 Nano-hardness of (a) worn disk surface (b) worn pin surface 3.2 SEM and EDX examination 

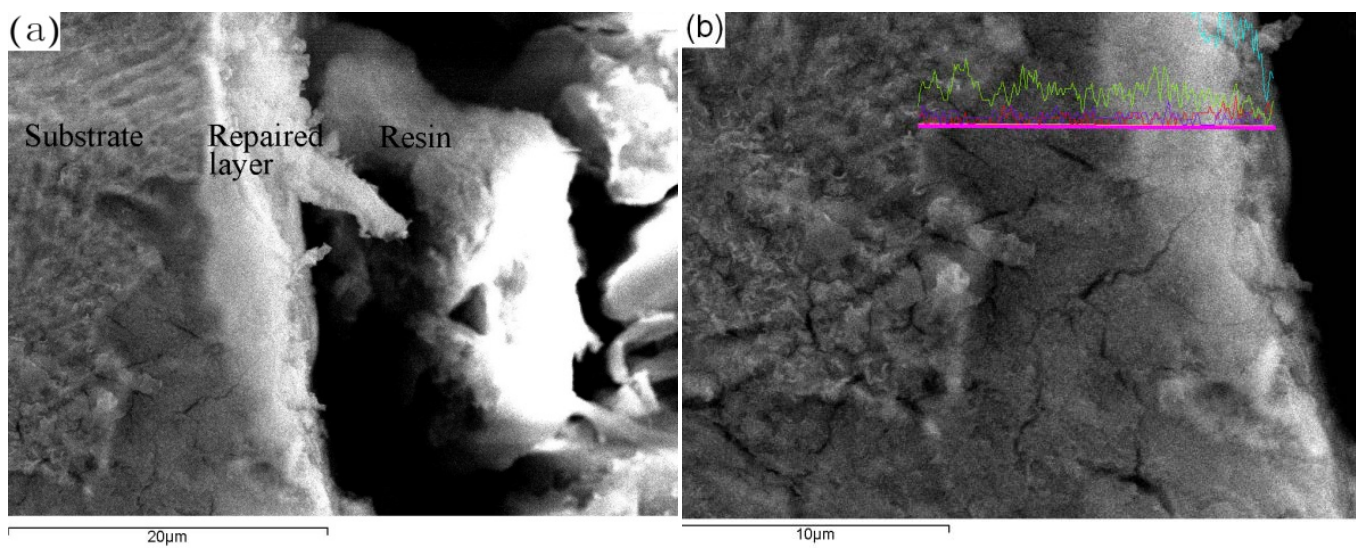

(c)

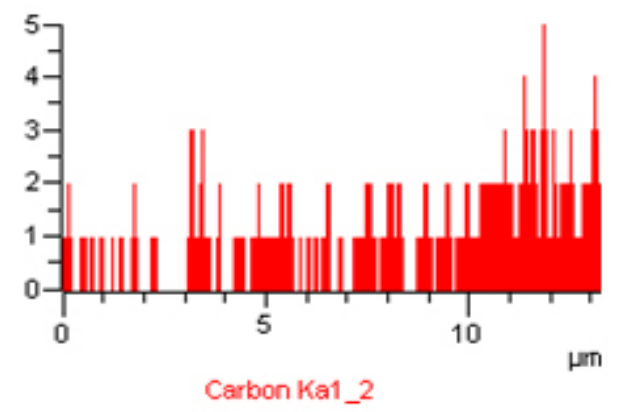

(e)

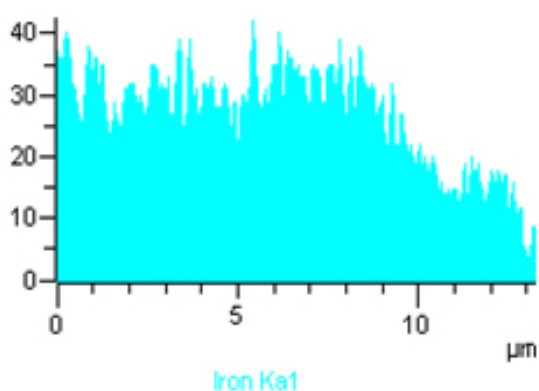

(d)

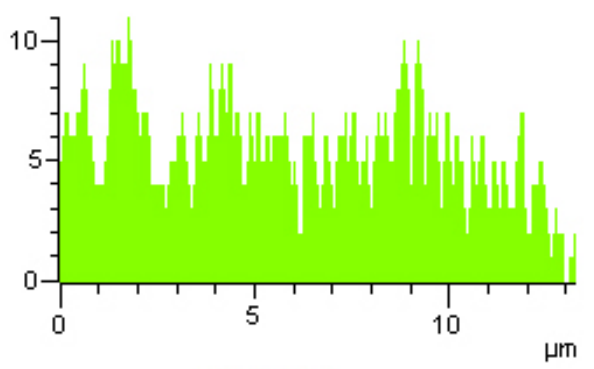

( $f$ )

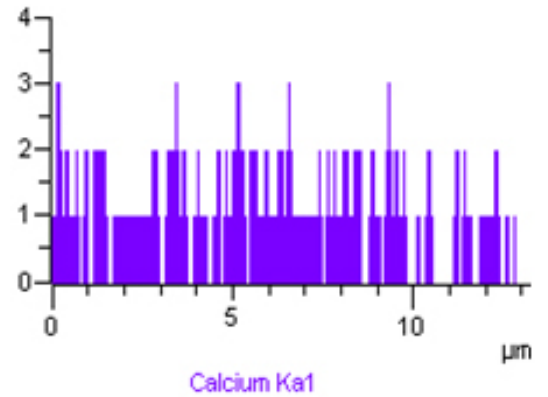

Fig. 2 Cross sectional SEM images and line scan spectra. (a) cross section view of the pin; (b) scan line position on the pin on a higher magnification view of the pin; (c) EDX data of carbon concentration variation along the scan line; (d) oxygen concentration variation; (e) iron concentration variation; (f) calcium concentration variation.

Fig. 2 shows the cross-sectional SEM images of the worn pin and line scan of several elements. Low-magnification SEM image of the cross section of the worn pin can be seen in Fig. 2a, a clear white and transparent repaired layer can be estimated to from 1 $\mu \mathrm{m}$ to $5 \mu \mathrm{m}$, the bottom of this cross section showed the smallest value of $1 \mu \mathrm{m}$. It is 
also noted that there is no obvious interface with the substrate, and crack was inhibited. The repaired layer of the worn pin is not even and the thickness is also different at the different worn areas, which demonstrated the repairing process is selective.

After it was amplified as Fig. 2b, line SEM/EDX scan was conducted along from the substrate to the repaired layer on the cross section of the pin. Fig. 2c-2f shows the EDX data of concentration of the element of carbon, oxygen, iron and calcium respectively. It can be seen that carbon concentration increased significantly on the area of the repaired layer, while iron concentration decreased with the distance from the substrate. High carbon content on the top surface would probably be diamond like carbon film. The DLC films are well known for their amorphous carbon microstructure, high hardness, low friction, and they show excellent wear resistance in oil-lubricated conditions. The hardness of the synthetic DLC films ranged from 10 to $60 \mathrm{GPa}[14,15]$, which is consistent to the hardness measured using nanoindentation as discussed in Section 3.1. The low friction coefficient and wear resistance had been verified in previous studies [10]. The detailed structural characterization of DLC films is carried out by using Raman spectroscopy as discussed in Section 3.3.

\subsection{Raman spectroscopy study}

Diamond-like carbon films have been widely studied for their excellent properties, such as high hardness [16], high wear resistance [16], low friction coefficient [17], chemical inertness, high electrical resistance and optical transparency in the visible and infrared lights [18]. DLC is a dense, metastable form of hydrogenated amorphous 
carbon a-C:H containing a significant $s p 3$ component. Structural characterization of a$\mathrm{C}: \mathrm{H}$ films has usually been carried out by using Raman spectroscopy. Generally, Raman spectra of DLC have been evaluated using the G-band around 1540-1580 $\mathrm{cm}^{-1}$ and D-band around $1350 \mathrm{~cm}^{-1}$. The G-band represents graphite and the D-band represents a disordered graphite-like structure (not diamond) [19].

It is determined that restoration layer could be DLC film from the characterization of the worn surface with transparency, smooth surface, high hardness, and remarkably high carbon concentration. In order to determine the existence of DLC films, Raman spectroscopy and XPS were used to carry out the structural characterization.

\subsubsection{Raman spectroscopy of the worn disk}

Fig. 3 shows the Raman spectra of restoration films formed on the worn disk in two different areas.
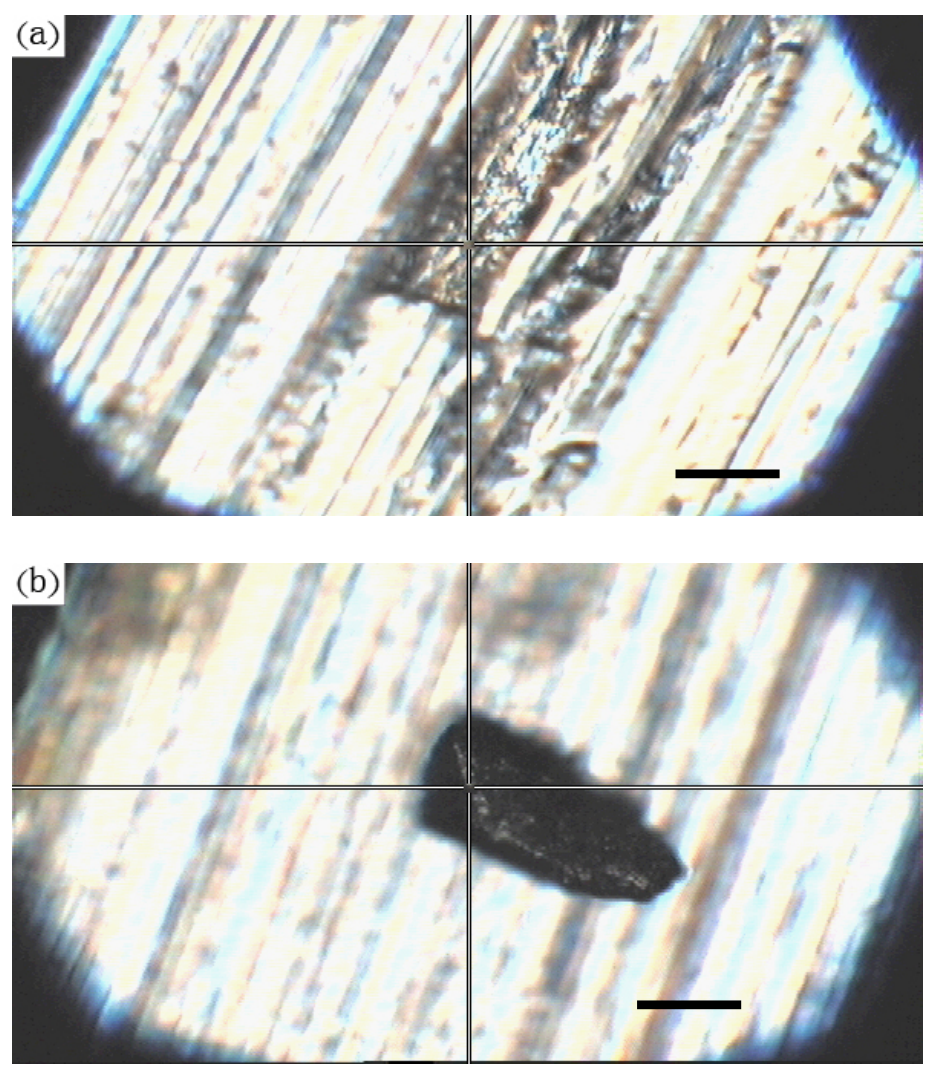


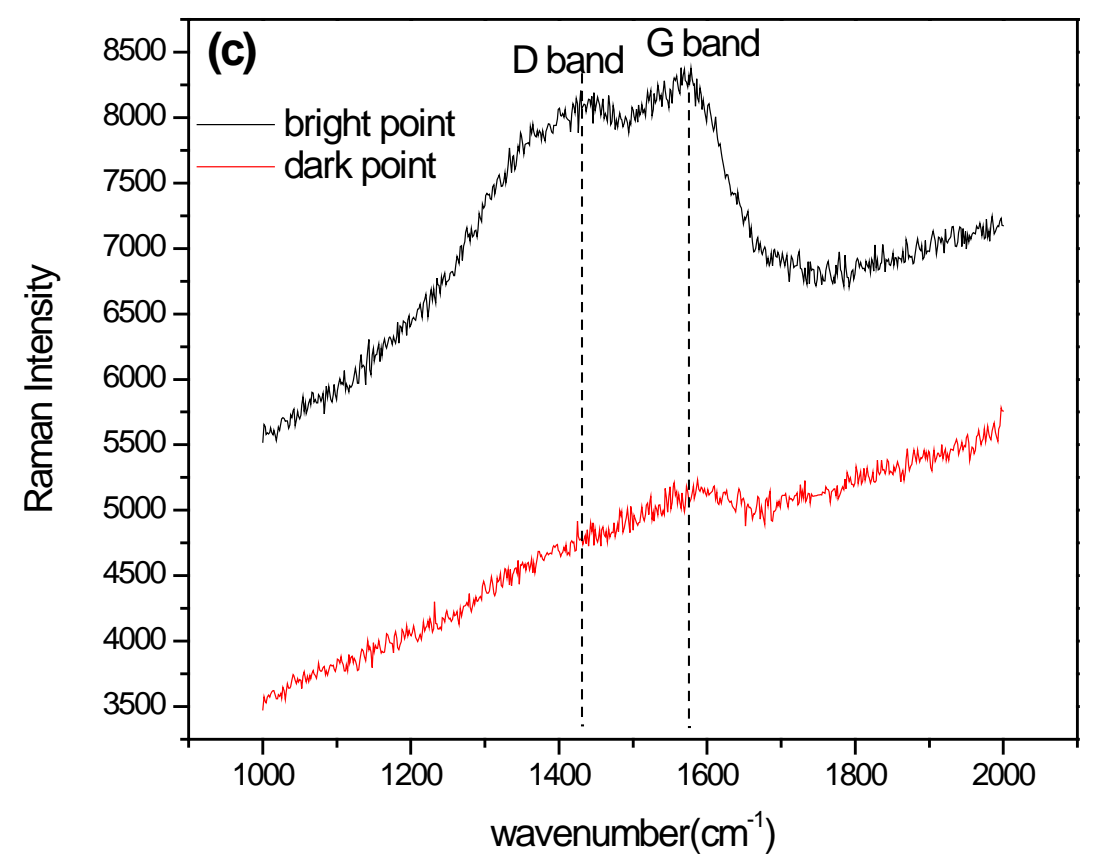

Fig. 3 Raman spectra for restoration film on the disk after 300-h test. Optical images of (a) bright area (b) dark area (c) Raman spectra. The scale bar in (a) and (b) is 100 $\mu \mathrm{m}$.

Two points in bright area (Fig. 3a) and dark area (Fig. 3b) were chosen to do Raman tests. As can be seen in Fig. 3c, the film formed on the disk has typical shape of DLC spectrum. Two points were selected to carry out the Raman spectroscopy test. For the dark point, the G-band located at $1564 \mathrm{~cm}^{-1}$ and the D-band appeared around 1426 $\mathrm{cm}^{-1}$. On the other hand, for the bright point, the G-band was weak and narrow in shape and located at $1578 \mathrm{~cm}^{-1}$. This suggested that the bright point has more of a graphite-like structure than other points. But this indicated that the restoration film is not even and thin, therefore, the G-band peak is not strong.

\subsubsection{Raman spectra of the worn pin}



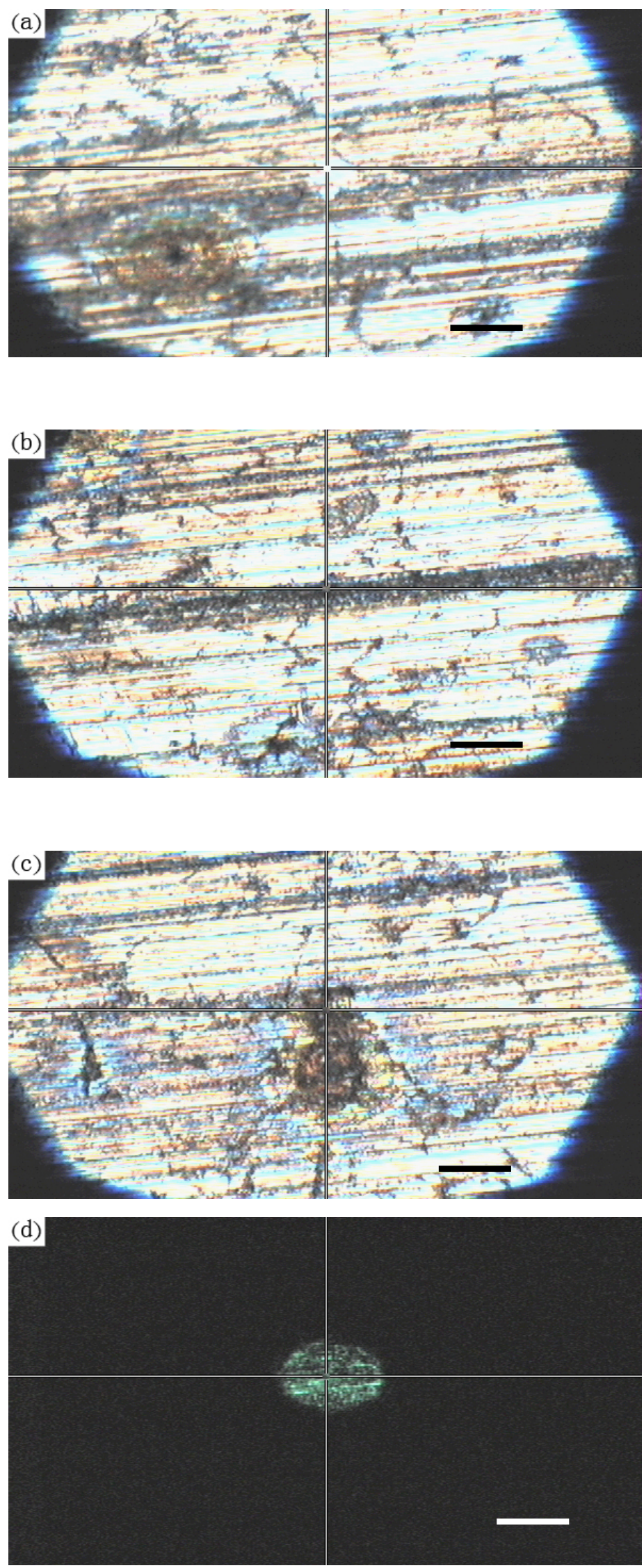


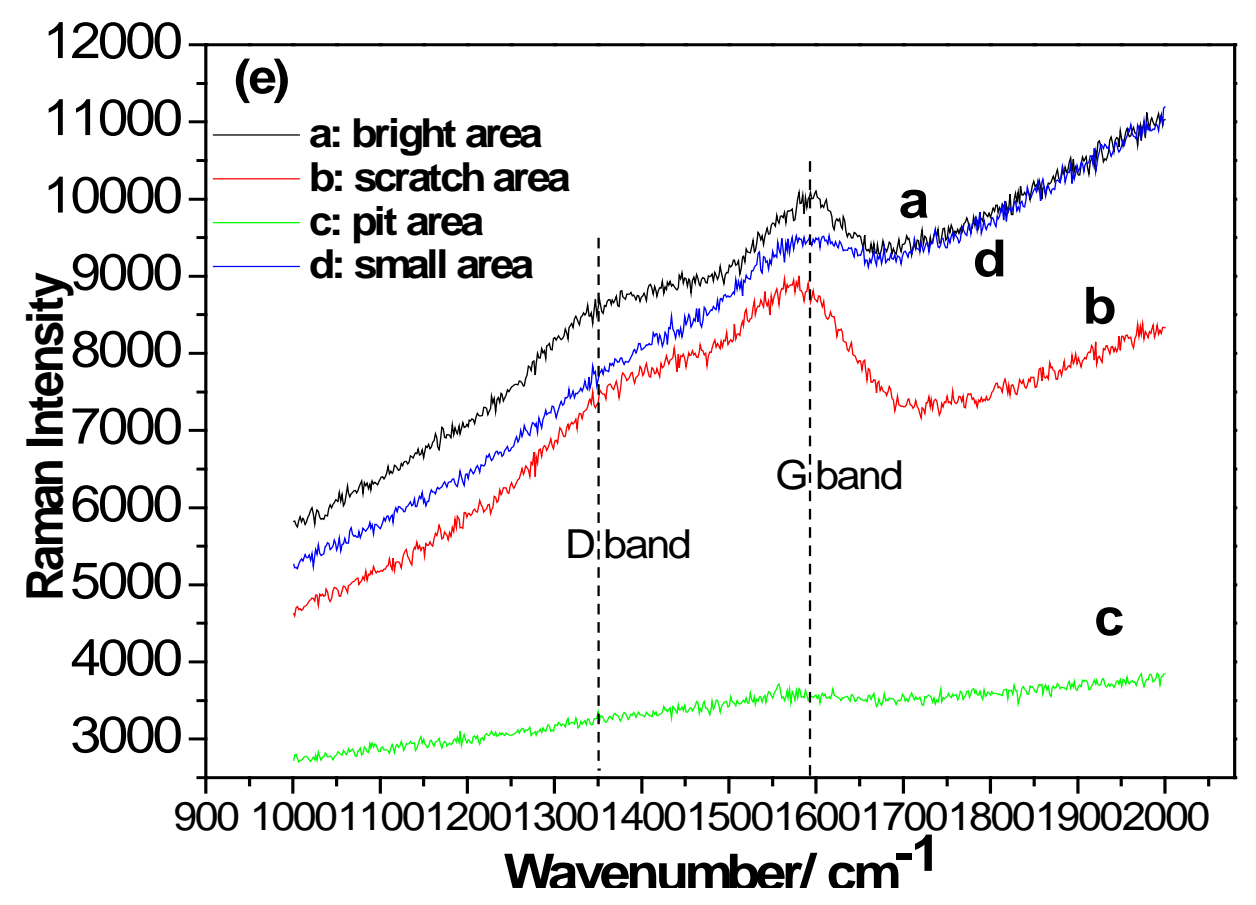

Fig. 4 Restoration films on the pin after 300-h test. Optical images of (a) bright area, (b) scratches area, (c) pit area, and (d) small area; (e) Raman spectra. The scale bar in (a)-(d) is $100 \mu \mathrm{m}$.

In view of thicker restoration layer of the pin during the friction and wear, the pin was also examined by using Raman spectroscopy test. Four areas (bright area, scratch area, pit area and small area) were selected to carry out the Raman spectroscopy test as shown in Fig.4 (a) - (d). Fig.4(e) shows the Raman spectra for restoration film on the pin after 300-h test. For the bright area in Fig. 4a, the G-band located at $1580 \mathrm{~cm}^{-1}$ and the D-band appeared around $1347 \mathrm{~cm}^{-1}$. For the scratch area in Fig. 4b, the Gband was weak and narrow in shape and located at $1580 \mathrm{~cm}^{-1}$ and the D-band appeared around $1405 \mathrm{~cm}^{-1}$.

For the pit area in Fig. 4c, no apparent G-band peak occurs. For the small area in Fig. 4d, the G-band located at $1580 \mathrm{~cm}^{-1}$ and no D-band. The spectrum shapes for the films at bright area, scratch area and a random small area are typical DLC shapes. 
In summary, most of the Raman spectra of the restoration layers in this study were consistent with a typical DLC Raman pattern [19]. The result verified the existence of DLC structure in the restoration layer.

\subsection{XPS structural characterizations}

Because XRD is not accurate to be used for amorphous structure, XPS analysis was carried out on the samples to determine its structural characterizations on the worn surface.

Fig. 5 shows XPS wide scan spectrum and high resolution XPS spectra for O 1s, C 1s, and Fe. The XPS wide scan spectrum indicates that the repaired layer on the pin appears to contain carbon, oxygen, iron, magnesium, silicon, calcium and zinc. It should be noted that the XPS spectrum shows the complexity of the engine oil system and the coexistence of different additives. Calcium and magnesium should be from detergents like calcium sulfonate, calcium phenate and magnesium sulfonate. Zinc is most likely from antiwear additives like ZDDP. While the nitrogen could be from dispersants, detergents, antioxidants or even friction modifiers.

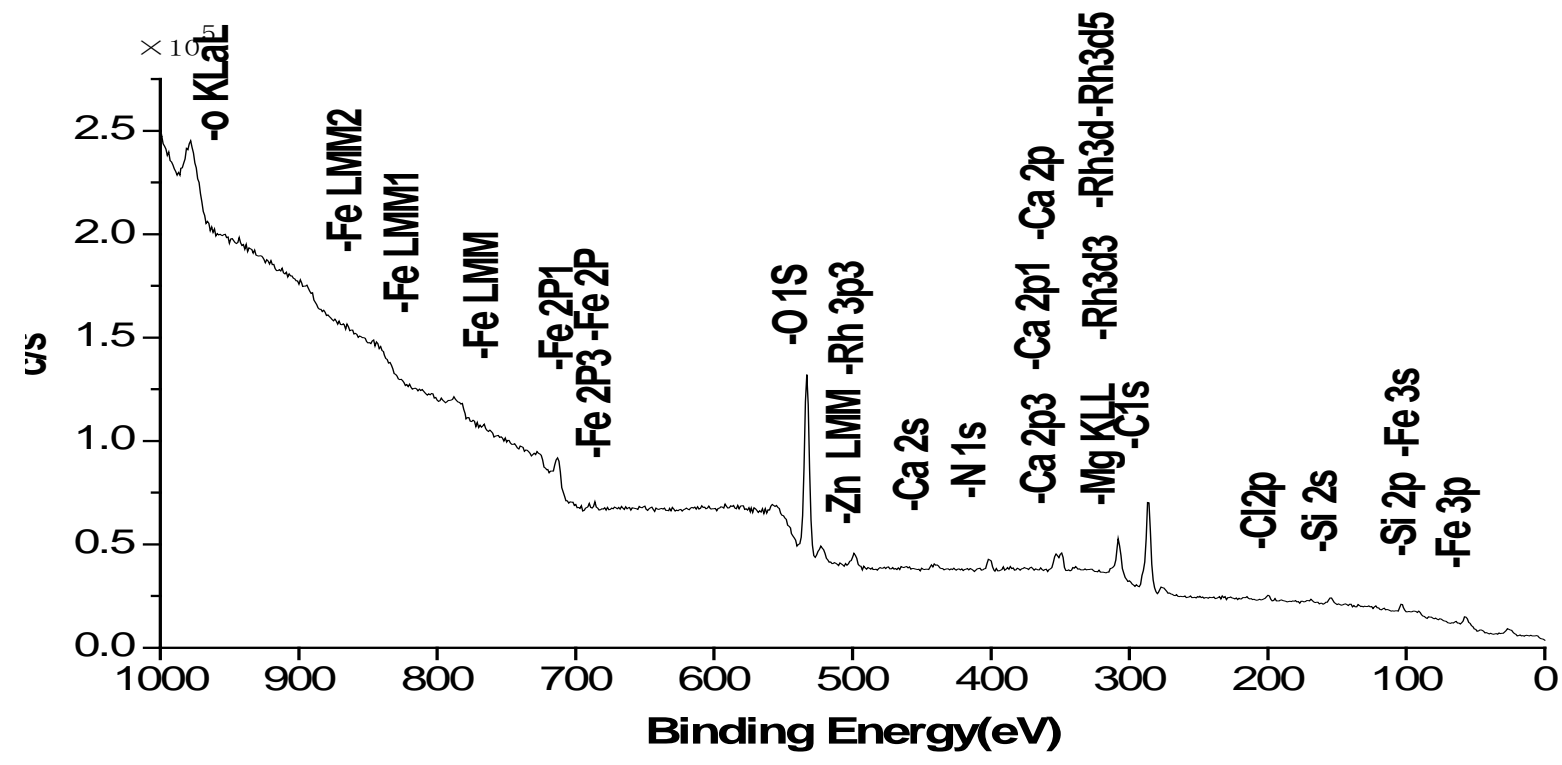


Fig. 5 XPS spectrum of the worn pin

From the XPS analysis, the contents of elements on the worn surface of the pin are: C 42.32 at \%,O 33.66 at \%, Mg 10.70 at \%, Fe 5.84 at \%, Si 3.87 at \%, Ca 2.42 at \%, and $\mathrm{Zn} 1.18$ at \%. A lot of carbon exists on the surface of the worn pin, and the value reached $42.32 \%$. This high carbon concentration on the worn pin surface is supposed to form DLC film, which was verified in Fig. 6.

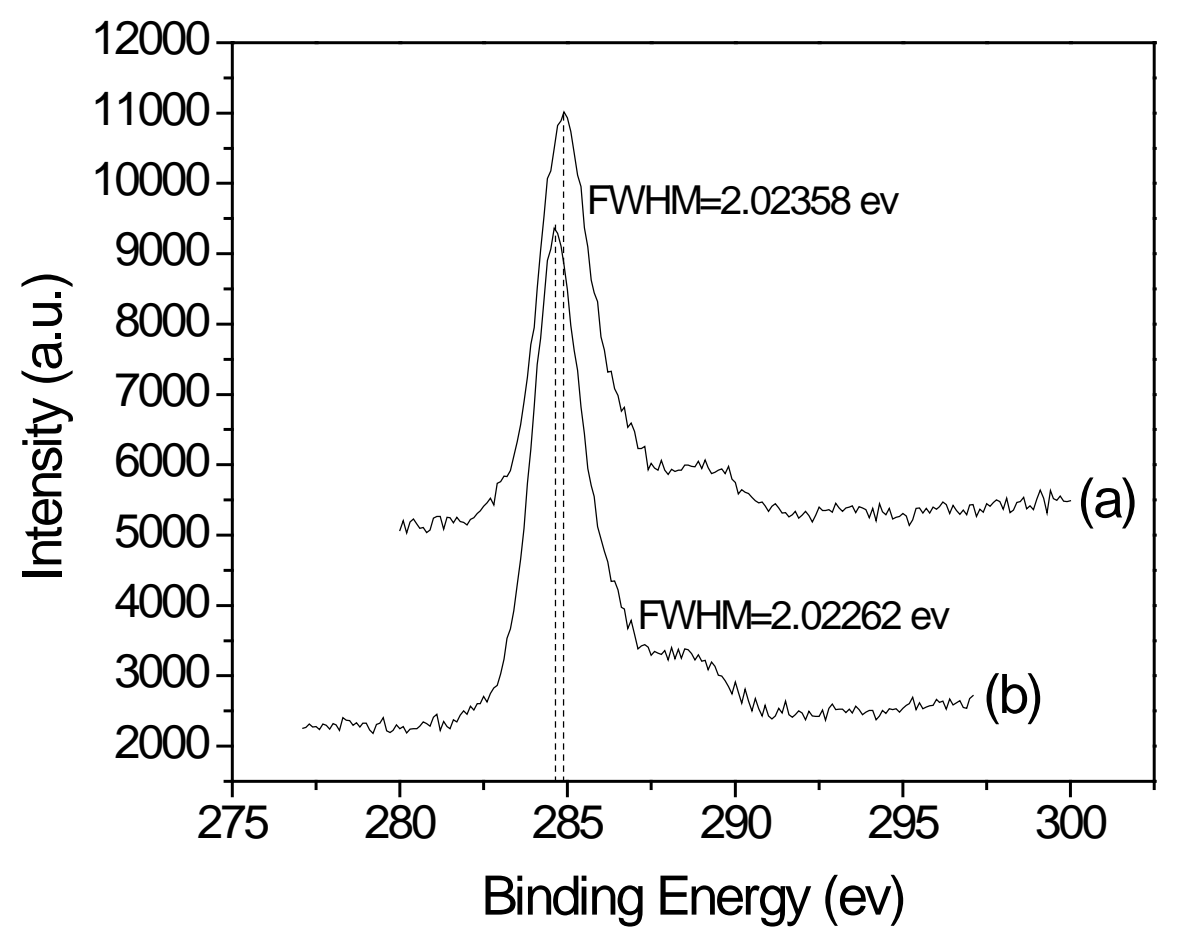

Fig. 6 XPS C 1s spectra for restoration films at the (a) worn pin (b) worn disk

As shown in Fig. 6, the $\mathrm{C}$ 1s peak position and the full width at half maximum (FWHM) were compared to those of diamond spectrum. In [20], the structures of the DLC films were analyzed by means of the peak positions and FWHMs of C $1 s$ spectra for the films, and $285.50 \mathrm{eV}$ and $284.15 \mathrm{eV}$ were adopted as the $\mathrm{C} 1 \mathrm{~s}$ peak position of 
the diamond component and the graphite component. The FWHM for graphite is 1.00 $\mathrm{eV}$, and the FWHM for diamond is $2.20 \mathrm{eV}$.

Fig. 6 shows C 1s spectra for DLC restoration film on the worn pin and worn disk. The peak positions for the DLC films formed on the pin and the disk shift from 284.9 eV(Fig. 6a) toward $284.6 \mathrm{eV}$ (Fig. 6b). Hence, the peak position approached that of diamond (285.50 eV). FWHM values for the restoration film on the worn pin and disk were $2.02358 \mathrm{eV}$ and $2.02262 \mathrm{eV}$ respectively, which also is close to that of diamond $(2.20 \mathrm{eV})$. Both measurements indicated that the restoration layer is DLC film.

Therefore, the structural characterization by XPS is consistent with that by Raman results.

\section{Discussions}

Approximately $1 \mu \mathrm{m}$ thick carbon-rich repaired layer was formed on the worn surface of the pin, but the SEM cross-sectional image showed its thickness was not uniform. This is why the nano-hardness of the worn surfaces was scattered. The carbon content of the 45 steel substrate was $0.42-\sim 0.50$ wt\%; however, XPS detected $42.32 \%$ carbon on the worn surface of the pin. Several ideas exist as to where the high-content carbon came from. It could be from carbon chain decomposition of the lubricating oil during the wear with the catalytic function of silicate particles. Raman analysis has indicated positively the restoration layer is DLC film.

Therefore, the mechanism of the silicate particles as the lubricating oil additive was more complicated than traditional extreme pressure additives [21, 22]. The mechanism of this advanced silicate additive was indicated by the following model. 
The complex physicochemical process could be divided into the five stages of superprecision grinding, energy activation, adhesion, partial restoration, and complete restoration film formation.

\section{(1) Super-precision grinding}

Micro morphology of friction pair contact surface is composed of micro convex and concave pits. Before the machine operates, concave pits are filled with lubricant and additive particles, as shown in Fig. 7a.

When the machine operates, a mechanical load forces the friction surface to be close.Micro convex points on the two surfaces penetrate into oil film, and collide and fracture, and then new abrasive particles get into oil. During a severe wear process, local temperature and pressure are remarkably large. Plastic deformation and fracture of a portion of micro concave would cause a rapid temperature increase, which leads the lubricant oil and additive to begin decomposition. After the additive is added, micro concaves function as the teeth of the grinding machine, and super-precisely grind the additive particles, breaking down the particles. Under high temperatures, micro metallurgical process occurs at the fracture section of micro concaves, as shown in Fig. $7 b$.

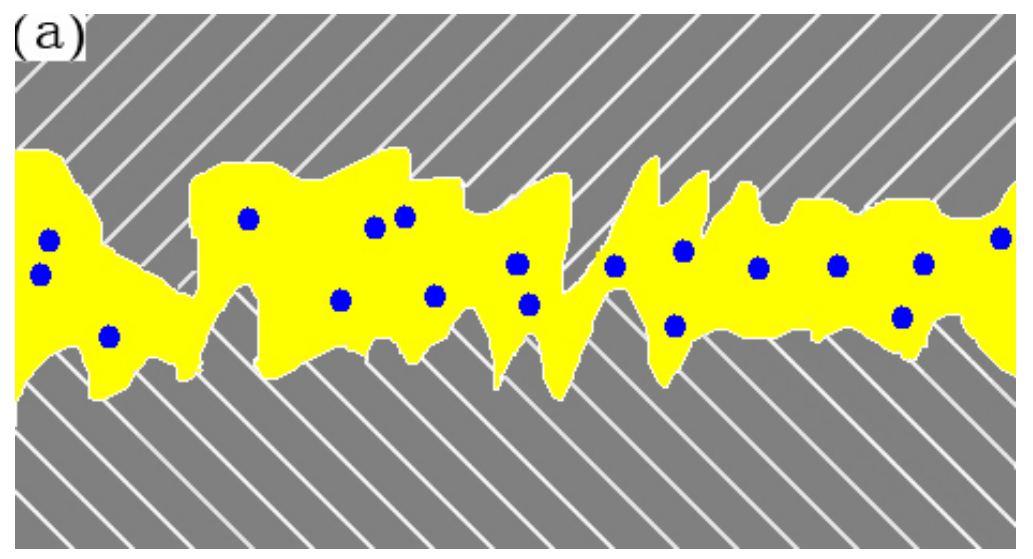




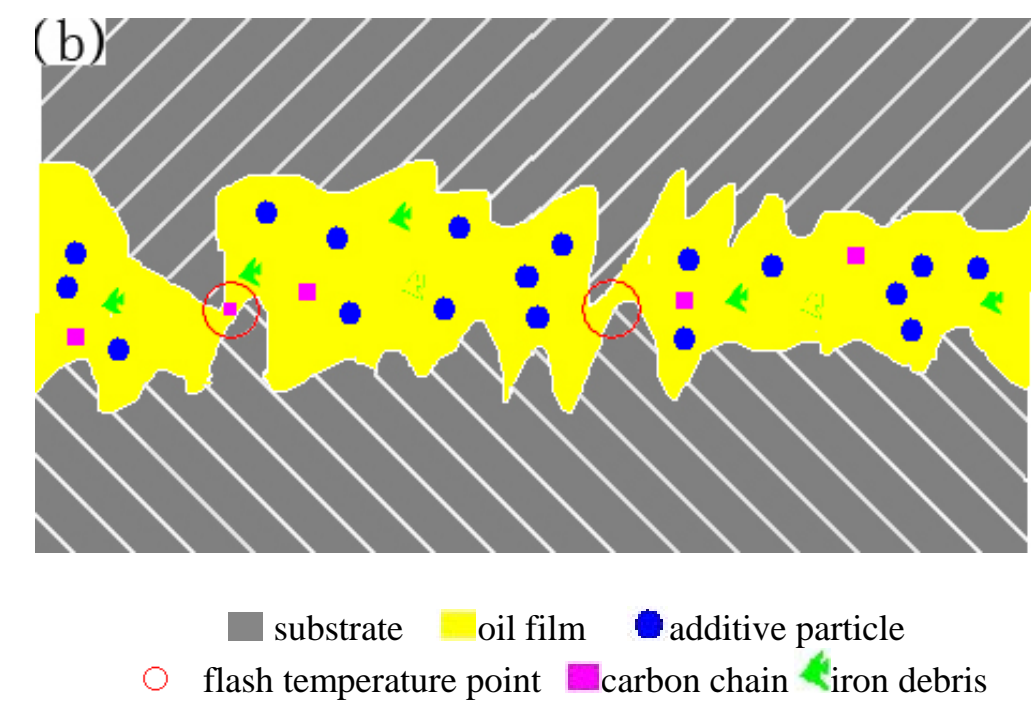

Fig. 7 Super-precision grinding process. (a) Concave pit is filled with lubricant and additive particles; (b) Under high temperatures, micro metallurgical process occurs at the fracture section of micro concaves

(2) Energy activation

With the machine running, lubricant oil decomposes gradually and the metal is ground gradually. Therefore, carbon chain and grinding debris are produced in the lubricant oil. These two situations may exist separately, or these two combine together, shown in Fig. 8a.

During high speed and high pressure friction and wear took place, and surface activity of carbon chain and iron debris was activated by active components of the additives, consequently, surface energy gradually increased, shown in Fig. 8b.

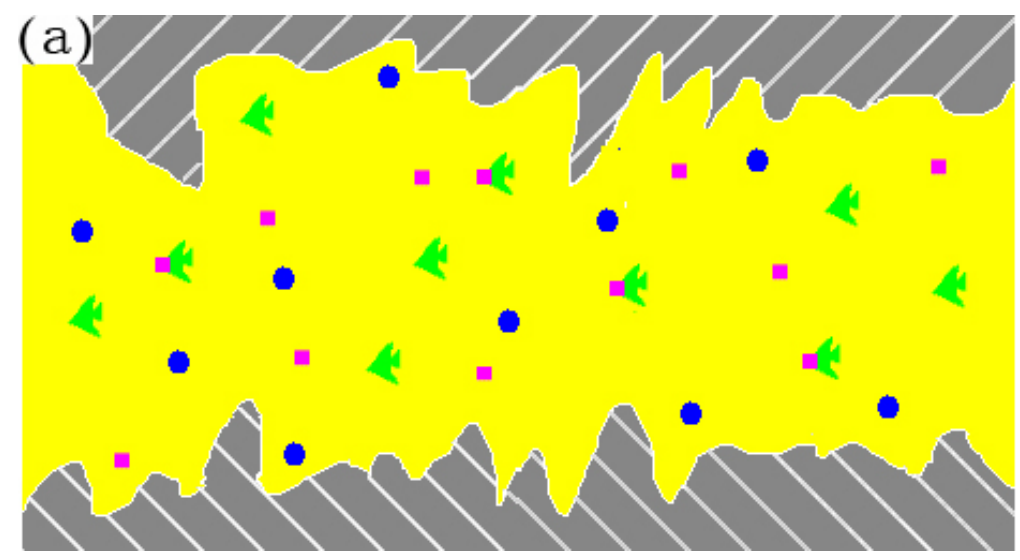




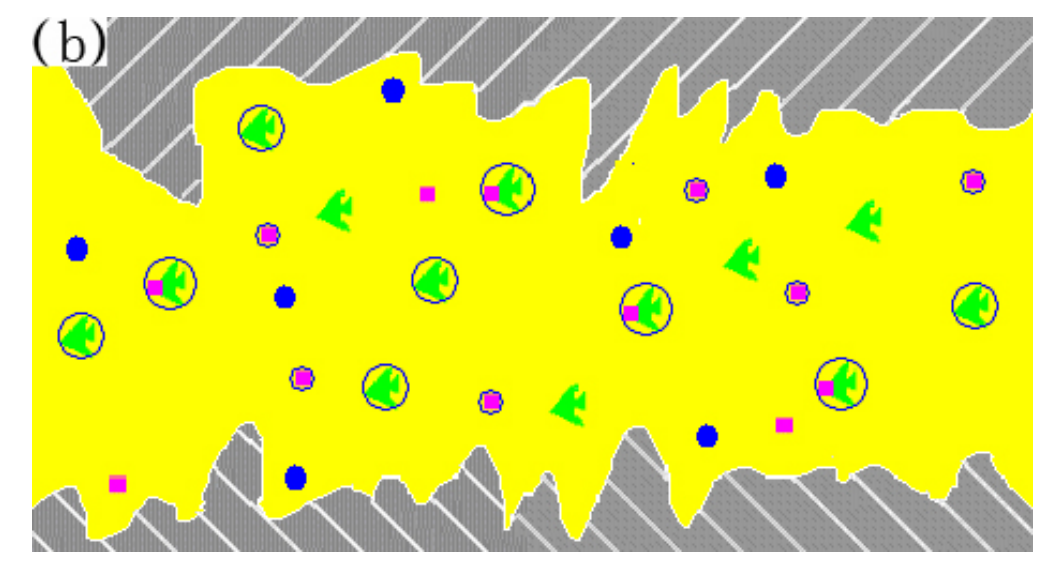

substrate oil film additive particle $\mathbf{a}$ carbon chain 4 iron debris $\checkmark$ the carbon chain activated by additive particles $D$ the iron debris activated by additive

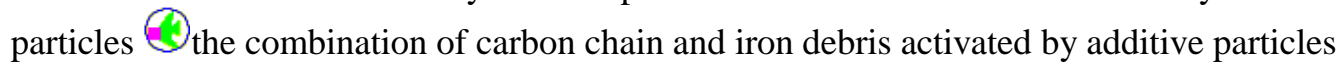

Fig. 8 Energy activation process. (a) The existence state of carbon chain and grinding debris; (b) Surface activity of carbon chain and iron debris is activated by active components of the additives

\section{(3) Adhesion}

Because of the friction, under local high temperature high pressure, carbon chain and iron debris are activated by additive particles move to the new surface and concave pits, and are adhered to the surface, as shown in Fig. 9a. With continuous friction running, a portion of particles goes into the lubricant oil, while another portion of particles is deposited on the surface of the substrate, as shown in Fig. 9b. For the concave pits and scratch sections, it is easier for the wear debris to be deposited and adhered, because friction occurs severely, causing more energy accumulation and adequate function of lubricant additive.

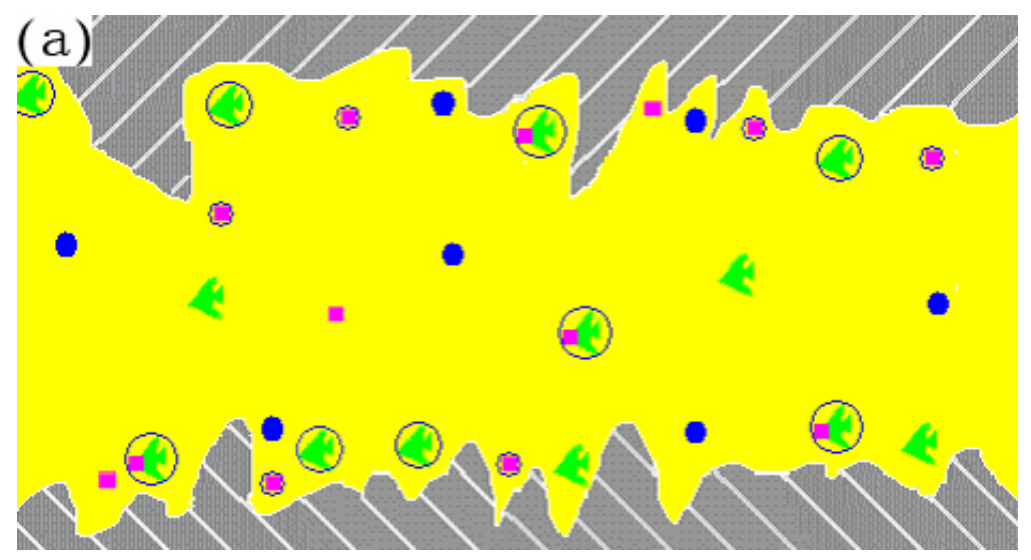




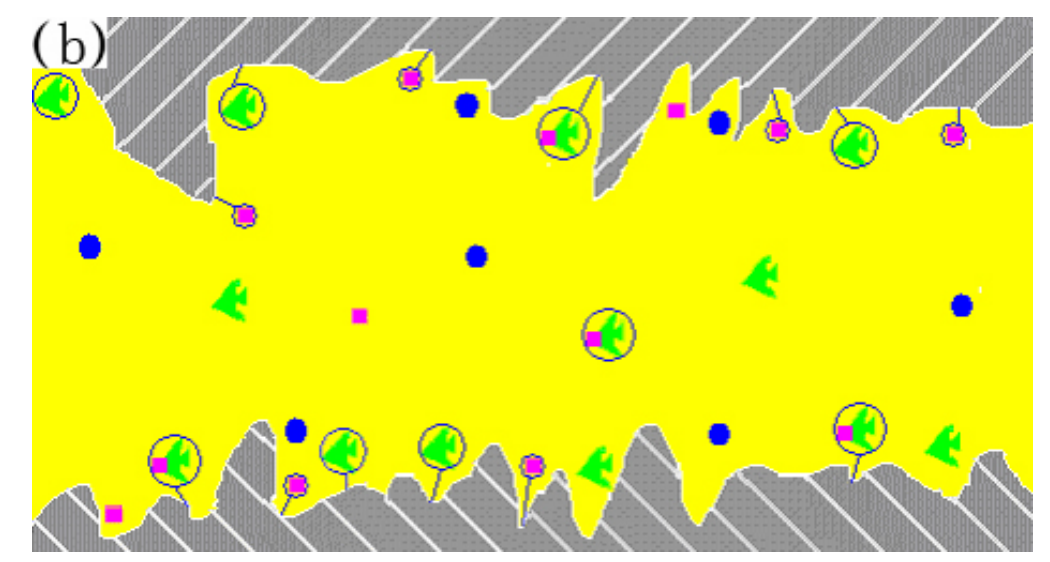

substrate oil film additive particle $\mathbf{a}$ carbon chain iron debris the carbon chain activated by additive particles the iron debris activated by additive particles $\vartheta_{\text {the combination of carbon chain and iron debris activated by additive particles }}$

Fig. 9 Adhesion process. (a) Carbon chain and iron debris moved to concave pit; (b) Some portion of particles was deposited on the surface of the substrate.

(4) Partial restoration

In continuous repetition of adhesion process, partial particles are deposited on the surface of partial concave pits and scratches. With the friction running, the energy accumulates continually. The deposited particles start to recombine in the structure, and finally partial restoration layer is formed, as shown in Fig. 10a. New surface is formed constantly as the friction process continues. The particles in the lubricant oil are deposited and partial restoration is constantly formed. Therefore, restoration film spreads out into the whole friction surface, as shown in Fig. 10b. For the convex section, the final restoration film is very thin because it is destroyed throughout the wear process. It can be concluded that the silicate additive possesses certain selectivity at different locations of the worn surface. Therefore, the thickness and distribution of the restoration film are not uniform throughout the interface. 

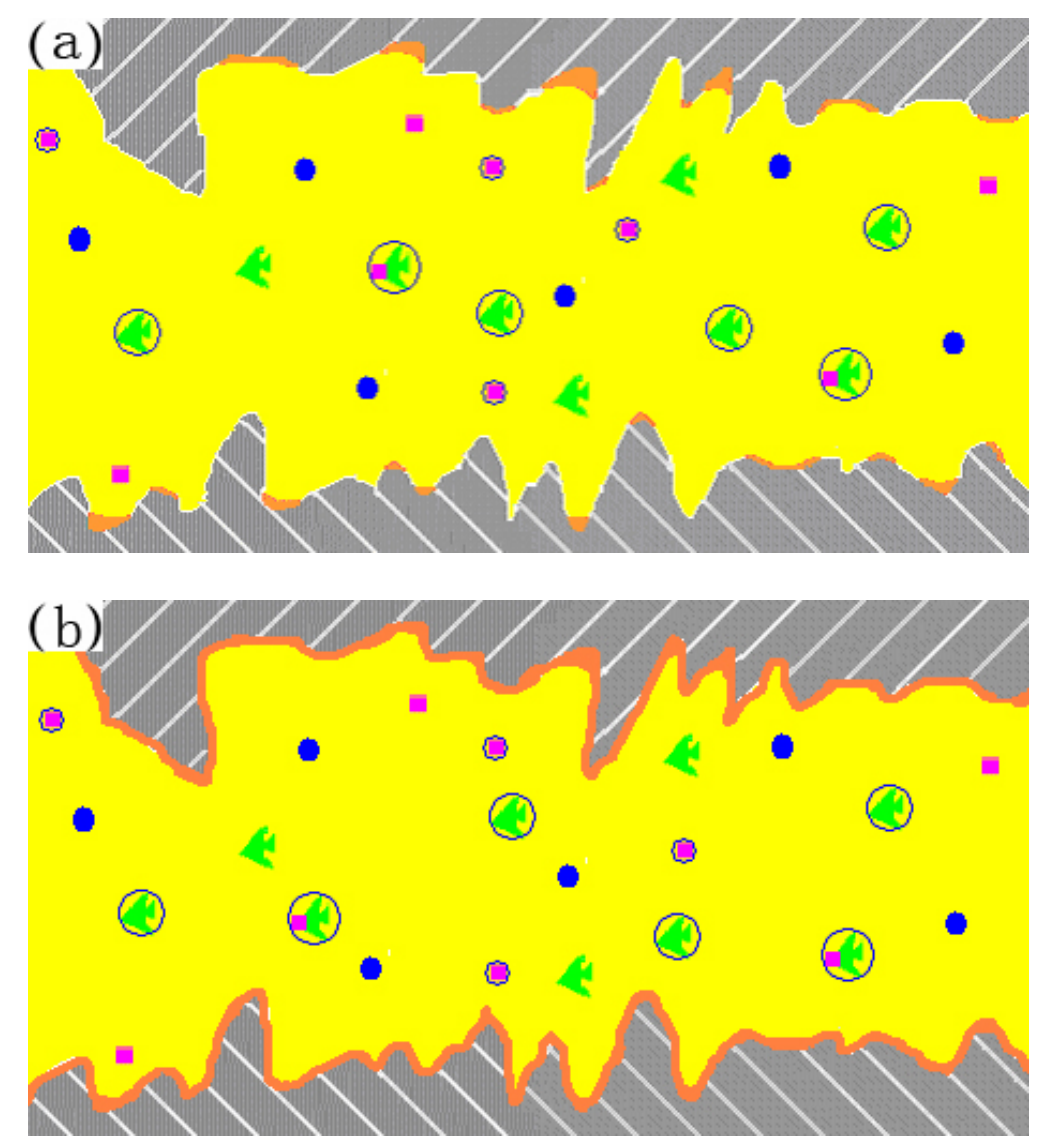

substrate oil film $\mathbf{c}$ additive particle $\mathbf{a}$ carbon chain 4 iron debris $\checkmark$ the carbon chain activated by additive particles the iron debris activated by additive

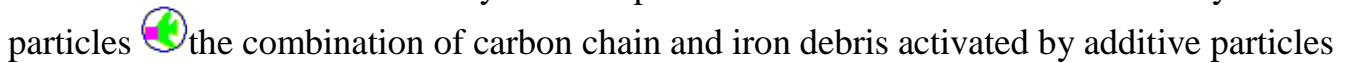

Fig. 10 Partial restoration process. (a) Deposited particles started to recombine in the structure, and small partial restoration layer was formed; (b) Restoration film spread out into the whole friction surface

(5) Formation of complete restoration layer

In ideal conditions, with the additive particles, the particles of the lubricant oil are deposited on the friction surface with high surface energy. If this process runs infinitely, a complete and thick restoration film would be formed on the friction surface, as shown in Fig. 11. 


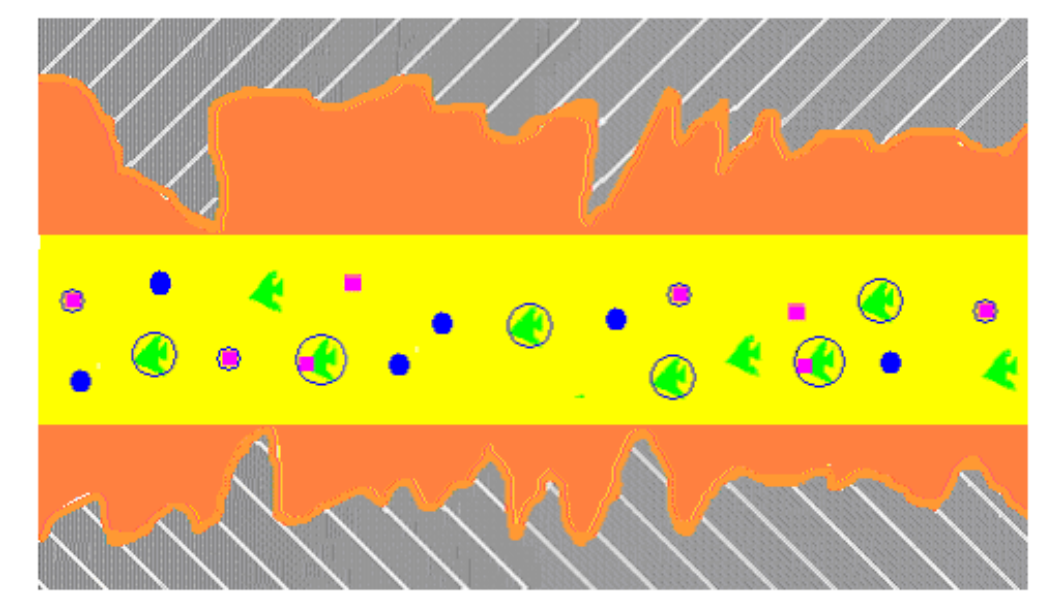
substrate oil film additive particle $\quad$ carbon chain iron debris
the carbon chain activated by additive particles the iron debris activated by additive particles the combination of carbon chain and iron debris activated by additive particles restoration film
Fig. 11 Formation of complete restoration layer

The excellent properties of the restoration film come from the structure recombination during the formation. A portion of carbon atoms are transferred into DLC structure under appropriate conditions, as verified by Raman analysis. It is fully indicated that under the catalytic function of the additive, carbon is accumulated and recombined on the friction surface, and then DLC film is formed.

\section{Conclusion and future work}

The following conclusions can be drawn from this study:

(1) The silicate-based lubricant additive showed a smooth surface with high nanohardness on the surface of the contact-pairs through forming a DLC layer.

(2) Structural characterizations of the restoration layer using Raman were performed. It was concluded that the worn disk and pin have a typical DLC shape, and the DLC film formed on the pin is more uniform and thicker than that on the disk.

(3) The mechanism of this advanced silicate-based lubricant additive was proposed as follows. The additive could act as a catalyst to promote a series of complex tribochemical reactions to form a DLC film on the worn surface of the contacts. 
The effective mechanism of the silicate-based lubricant additive could be the silicate additive improved the activities of atoms on the worn surface, absorbed carbon chain broken in the lubricating oil, captured a part of iron debris, and implemented recombination on the worn surface, thus DLC was formed.

The future work includes using additive-free base oil not engine oil, because it will eliminate the effect of other additives in the system. Additionally, the amorphous carbon structures can be further analyzed by using transmission electron microscopy. 


\section{References}

[1] L. A. Huezo, Kotvis, P. V., Crumer, C., Soto, C., Tysoe, W. T., "Surface chemistry and extreme pressure lubricant additive properties of chloroform on iron," Applied Surface Science, vol. 78, pp. 113-122, 1994.

[2] H. Spikes, "Tribology research in the twenty-first century," Tribology International, vol. 34, pp. 789-799, 2001.

[3] B. Yu, Liu, Z.L., Zhou, F., Liu, W.M., Liang, Y.M., "A novel lubricant additive based on carbon nanotubes for ionic liquids," Materials Letters, vol. 62, pp. 2967-2969, 2008.

[4] H. L. Yu, Xu, Y., Shi, P. J., Wang, H. M., Zhao, Y., "Tribological behaviors of surface-coated serpentine ultrafine powders as lubricant additive," Tribology International, vol. 43, pp. 667675, 2010.

[5] H. L. Yu, Xu, Y., Shi, P.J., Xu, B.S., Wang, X.L., "Tribological properties and lubricating mechanisms of $\mathrm{Cu}$ nanoparticles in lubricant," Transactions of Nonferrous Metals Society of China, vol. 18, pp. 636-641, 2008.

[6] A. S. Nikolaevich, "Method of Treatment of Friction Surfaces of Friction Units," UA Patent, 2001-5-31.

[7] A. S. Nikolaevich, "Method of Treatment of Friction Surfaces of Friction Units," UA Patent, 2001-10-10.

[8] J. J. Liu, and Guo, F.W., "The application and analysis of a new self-repairing technology on the frictional surface," China Surface Engineering, vol. 66, p. 4, 2004.

[9] J. Xu, Wang, F., and Liu, Z.L., "Investigation of the tribology behaviors of auto-restoration additive under heavy loading conditions.," Surface Review and Letters, vol. 14, pp. 329-334, 2007.

[10] W. Yue, Wang, C.B., and Tian, B., "Effect of ceramic additive in lubricating oil on contact fatigue and wear performance of steel/steel friction pair," Transactions of Materials and Heat Treatment, vol. 27, p. 6, 2006.

[11] Y. Yu, Gu, J.L., and Kang, F.Y., "Surface restoration induced by lubricant additive of natural minerals," Applied Surface Science, vol. 253, pp. 7549-7553, 2007.

[12] J. Zhang, B. Tian, and C. Wang, "Long-term surface restoration effect introduced by advanced silicate based lubricant additive," Tribology International, vol. 57, pp. 31-37, 2013.

[13] L. Wu, X. Guo, and J. Zhang, "Abrasive Resistant Coatings-A Review," Lubricants, vol. 2, pp. 66-89, 2014.

[14] J. S. Wang, Sugimura, Y., Evans, A. G., and Tredway, W. K., "The mechanical performance of DLC films on steel substrates," Thin Solid Films, vol. 325, pp. 163-174, 1998.

[15] C. Ziebert, Rinke, M., Stuber, M., Ulrich, S., and Holleck, H., "Interfaces and temperature stability of stepwise graded DLC films studied by nanoindentation and Raman spectroscopy," Surface and Coatings Technology, vol. 200, pp. 1127-1131, 2005.

[16] A. Grill, "Tribology of diamondlike carbon and related materials: an updated review," Surface and Coatings Technology, vol. 94-95, pp. 507-513, 1997.

[17] H. Dimigen, and Klages, C.P., "Microstructure and wear behavior of metal-containing diamond-like coatings," Surface and Coatings Technology, vol. 49, pp. 543-547, 1991.

[18] N. Paik, "Raman and XPS studies of DLC films prepared by a magnetron sputter-type negative ion source," Surface and Coatings Technology, vol. 200, pp. 2170-2174, 2005.

[19] N. Yamauchi, Demizu, K., Ueda, N., Cuong, N. K., and Sone, T., "Friction and wear of DLC films on magnesium alloy," Surface and Coatings Technology, vol. 193, pp. 277-282, 2005.

[20] Y. Taki, and Takai, O., "XPS structural characterization of hydrogenated amorphous carbon thin films prepared by shielded arc ion plating," Thin Solid Films, vol. 316, pp. 45-50, 1998.

[21] J. F. Lin, Shih, M.G., Chen, Y.W., "The tribological performance of 6061 aluminum alloy/graphite composite materials in oil lubrications with EP additives," Wear, vol. 198, pp. 58-70, 1996.

[22] Y. Wan, Xue, Q.J., "Friction and wear characteristics of p-containing antiwear and extreme pressure additives in the sliding of steel against aluminum alloy," Wear, vol. 188, pp. 27-32, 1995. 


\section{List of Figures}

Fig. 1 Nano-hardness of (a) worn disk surface (b) worn pin surface

Fig. 2 Cross sectional SEM images and line scan spectra. (a) cross section of the pin (lower magnification) (b) line scan position on the cross section of the pin (higher magnification) (c) carbon concentration variation (d) oxygen concentration variation; (e) iron concentration variation (f) calcium concentration variation

Fig. 3 Raman spectra for restoration film on the disk after 300-h test. (a) bright area (b) dark area (c) raman spectra. The scale bar in (a) and (b) is $100 \mu \mathrm{m}$

Fig. 4 Raman spectra for restoration film on the pin after 300-h test. (a) bright area (b) scratches area (c) pit area (d) small area (e) raman spectra. The scale bar in (a)-(d) is $100 \mu \mathrm{m}$

Fig. 5 XPS spectra of the worn pin

Fig. 6 XPS C 1s spectra for restoration films at the (a) worn pin (b) worn disk

Fig. 7 Superprecision grinding. (a) Concave pit is filled with lubricant and additive particles; (b) Under the high flash temperature, micro metallurgical process occurred at the fracture section of micro concaves

Fig. 8 Energy activation. (a) The existence state of carbon chain and grinding debris; (b) Surface activity of carbon chain and iron debris was activated by active components of the additives

Fig. 9 Adhesion. (a) Carbon chain and iron debris moved to concave pit; (b) Some portion of particles were deposited on the surface of the substrate

Fig. 10 Partial restoration. (a) Deposited particles started to recombine in the structure, and small partial restoration layer was formed; (b) Restoration film spread out into the whole friction surface

Fig. 11 Formation of complete restoration layer 